\title{
Translational research: Is there a future?
}

\author{
Edward Donald Verrier, MD
}

See related article on page 1604.

From the Division of Cardiothoracic Surgery, University of Washington, Seattle, Wash.

Received for publication Jan 12, 2007; accepted for publication Jan 25, 2007.

Address for reprints: Edward Donald Verrier, MD, University of Washington, Surgery, 1959 NE Pacific St, Box 356310, Seattle, WA 98195.

J Thorac Cardiovasc Surg 2007;133:1409-11 $0022-5223 / \$ 32.00$

Copyright (C) 2007 by The American Association for Thoracic Surgery

doi:10.1016/j.jtcvs.2007.01.048
$\mathrm{I}$ $\mathrm{n}$ this issue of the Journal, Tardif and associates ${ }^{1}$ from the Montreal Heart Institute report the results of the double-blind, placebo-controlled MC-1 (cardoxal) to Eliminate Necrosis Damage in Coronary Artery Bypass Grafting (MEND-CABG) trial conducted at 40 centers in the United States and Canada between April 2004 and July 2005. The MEND-CABG trial is a phase II study intended to evaluate the potential cardioprotective and neuroprotective effects of pyridoxal-5' -phosphate (cardoxal) in patients undergoing high-risk coronary artery bypass grafting (CABG). Pyridoxal-5' -phosphate monohydrate (P-5'-P, or MC-1) is a naturally occurring metabolite of pyridoxine that acts as a purinergic receptor antagonist that blocks intracellular influx of calcium, thereby theoretically reducing cell damage during episodes of ischemia and reperfusion.

It is important to realize that in the study design the authors and their statistical colleagues decided on the primary and secondary end points on the basis of a careful account of predictable event rates that would balance enrollment (number and associated costs) with need to achieve believable statistical significance. The primary efficacy end point was the combined incidence of cardiovascular death, nonfatal myocardial infarction, and nonfatal cerebral infarction to day 30. Secondary efficacy end points included individual components of the combined end point, area under the curve of creatine kinase isoenzyme MB (CK-MB) within the first 24 hours after $\mathrm{CABG}$, mortality from all causes, and composite and component end points to postoperative day 90 . Myocardial infarction was defined in the independent central core electrocardiographic laboratory as follows: (1) peak CK-MB value greater than $50 \mathrm{ng} / \mathrm{mL}$, or greater than $35 \mathrm{ng} / \mathrm{mL}$ with electrocardiographic evidence of Q waves through postoperative day 4; (2) peak CK-MB greater than $25 \mathrm{ng} / \mathrm{mL}$ or new $\mathrm{Q}$ waves occurring after postoperative day 4; and (3) Q-wave or non-Q wave myocardial infarction identified by investigator and confirmed by central core laboratory.

P-5'-P did not significantly affect relative to placebo the prespecified composite or component end points of cardiovascular death, myocardial infarction, and nonfatal cerebral infarction at either low $(250 \mathrm{mg} / \mathrm{d}$ for 30 days $)$ or high $(750 \mathrm{mg} / \mathrm{d}$ for 30 days) doses. In a post hoc analysis in which the peak CK-MB level was increased to $70 \mathrm{ng} / \mathrm{mL}$ or $100 \mathrm{ng} / \mathrm{mL}$, there were significant reductions in the combined end points of death, nonfatal stroke, and nonfatal myocardial infarction with both dosing regimens $(250$ and $750 \mathrm{mg} / \mathrm{d})$. On the basis of this post hoc analysis, the authors conclude, "Cardoxal may be particularly effective in preventing larger, more significant perioperative infarcts." They then suggest that a larger, better powered trial is needed to evaluate further the cardioprotective effects of cardoxal.

There are a number of aspects of this trial that deserve comment. First, we currently live in a clinical world in which more and more emphasis is placed on evidence-based medicine. In the pyramid of importance of clinically relevant literature, systematic reviews with meta-analysis are at the top of the pyramid, followed by randomized, controlled, double-blind studies (followed by cohort studies, case controlled series, case series, case reports, ideas, editorials, opinions, animal research, and in vitro research). ${ }^{2}$ As surgeons, we are often criticized by our medical colleagues for our lack of scientific credibility, because so much of our reported literature revolves around retrospective case series. For that reason, publishing this type of well-designed, well-conducted, and well-reported trial is important even if the results are essentially negative or only suggestive. 
Second, we must be extremely careful in drawing any meaningful conclusions related to efficacy or accuracy in such a study from a post hoc analysis of the data as suggested in the article. Numerous articles in the literature describe erroneous statistical conclusions on the basis of reanalysis of the raw data or misrepresentation of the a priori assumptions of a trial. ${ }^{3,4}$ Such post hoc analyses are problematic because they are undertaken after review of the trial results. The clinical data set is then divided into significantly different subgroups, and the statistical corrections usually do not account for these additional analyses. Whenever we increase the number of comparisons, some statistical adjustment is necessary, such as a Bonferroni or Hochberg correction ${ }^{5}$; otherwise, there is a much greater chance of incurring a type I (or $\alpha$ ) error (probability of incorrectly rejecting the null hypothesis) or a type II (or $\beta$ ) error (probability of incorrectly accepting the null hypothesis). This then would require $P$ values considerably lower than .05 to be of any real value in any later analysis. In the post hoc analysis of the primary end points in this trial at the peak CK-MB levels of 70 or $100 \mathrm{ng} / \mathrm{mL}$, the $P$ values were between .03 and .15 , depending on the dose regimen. For this reason, the results and analysis of the MEND study must be interpreted as being negative, and any meaningful post hoc analysis must be viewed with a degree of skepticism.

This study can easily be compared with the recently published Pexelizumab for Reduction of Infarction and Mortality in Coronary Artery Bypass Grafting Surgery (PRIMO-CABG) study, which looked at the impact of complement inhibition (with a monoclonal antibody to the C5a complement complex called pexelizumab) during $\mathrm{CABG}{ }^{6}$ When the PRIMO-CABG phase 3 study was published in 2004, the primary end point was a reduction in the composite end point of death or myocardial infarction. The PRIMOCABG trial was similar to the MEND-CABG trial in that the a priori expected primary end point for PRIMO-CABG was also not achieved. In a post hoc analysis, the PRIMOCABG investigators increased the CK-MB analysis to 75 $\mathrm{ng} / \mathrm{mL}$ (as is now being done in the MEND-CABG trial) and found a high-risk group of patients with more than two risk factors who showed a "highly significant" benefit for both the primary and secondary end points. The PRIMOCABG investigators then went on to a second phase 3 trial, as requested by the Food and Drug Administration, enrolling more than 4000 additional patients worldwide. In this second phase 3 trial, the investigators redesigned the trial with the more restrictive primary and secondary end points suggested in the first phase 3 publication. Absolutely no efficacy or statistical significance was obtained in the final study, and the drug was abandoned (unpublished data). Similarly, a different preliminary phase 3 trial of pexelizumab during acute coronary interventions was suggestive of benefit after an initial post hoc analysis; however, the subsequent, definitive phase 3 trial was recently reported, and once again the drug was completely nonefficacious. ${ }^{7}$ I strongly suspect that any further investigation looking at cardoxal will follow a similar pattern and yield statistically insignificant results.

The final question relates to why promising preliminary studies in animals do not translate into similar clinical results in human beings. Clearly, inflammation has been identified in the last 20 years as a major pathophysiologic mediator of sepsis, atherosclerosis, and ischemia-reperfusion syndromes. This has led to a huge basic science literature looking at molecular and cellular mechanisms of reperfusion injury, microvascular integrity, metabolic tolerance, coagulation abnormalities, and inflammatory damage. ${ }^{8}$ What is most interesting is the almost total lack of efficacy seen in clinical trials attempting to translate the basic science into clinical practice. ${ }^{6,7}$ The obvious conclusion is that we have made little or no clinical advance in translational research in trauma, sepsis, atherosclerosis, or any ischemia-reperfusion state. ${ }^{9}$ The obvious question, then, is why? Certainly, the inflammatory and coagulation cascades as we now understand them are highly complex, interconnected pathways, networks, and molecular systems that are teleologically redundant as a result of eons and eons of inflammatory challenge and genetic evolution. What may be more important are concepts that are now based on recent understandings of human genomics and proteonomics. ${ }^{10}$ Variable responses to pharmacologic agents seem to have a more complex origin involving the interplay between multiple genetic factors (genes and their products) and nongenetic factors (environmental influences). We now understand that genetic variations in the human population, including single-nucleotide polymorphisms, small deletions and insertions, and other structural differences (including haplotypes, sets of genes inherited as units) are very common and may be critical to our understanding of the relationships between genotype and biologic function. Such genetic variation may account for the tremendous clinical variation and how human beings respond to various similar insults such as ischemia-reperfusion, sepsis, and shock. It may also explain why it is so difficult to find efficacy in altering a single cellular or molecular target. Simply targeting a single receptor at the beginning of the inflammatory cascade or targeting an upregulated new protein at the end of the cascade, which might alter the cellular phenotype, seems increasingly unlikely to be efficacious. Whether multiple targets will be synergistic, leading to a predictable clinical benefit, or whether there may be critical central control points within the cellular communication pathways (such as signal transduction pathways or transcriptional mediators) remains to be determined. Genomics may provide new insights into why we see such variability in 
responses to insults and to new therapy and why there are such different physiologic responses in different species.

Looking beyond the well-presented results of this study will yield insights for all of us as we look to the future of translational research. If we are to make cardiopulmonary bypass and cardiothoracic and vascular surgery safer and improve outcomes, we will have to look beyond technology and understand science. Certainly studies such as the one published by the MEND-CABG group should stimulate all surgeons to look at the critical importance of basic science to our increasingly complex clinical challenges.

\section{References}

1. Tardif JC, Carrier M, Kandzari DE, Emery R, Cote R, Heinonen T, et al. Effects of pyridoxal-51-phosphate (cardoxal) in patients undergoing high-risk coronary artery bypass surgery: results of the MENDCABG randomized study. J Thorac Cardiovasc Surg. 2007;133:160411.

2. Sackett DL, Straus S, Richardson S, Rosenberg W, Hopkins RB. Evidence based medicine: how to practice and teach. 2nd ed. London: Churchill-Livingston; 2000.
3. Goodin DS. Disease-modifying therapy in MS: a critical review of the literature. Part I: analysis of clinical trial errors. J Neurol. 2004;251 Suppl 5:v3-v11.

4. Sankoh AJ, Huque MF, Dubey SD. Some comments on frequently used multiple endpoint adjustment methods in clinical trials. Stat Med. 1997;16:2529-42.

5. Hochberg Y, Benjamini Y. More powerful procedures for multiple significance testing. Stat Med. 1990;9:811-8.

6. Verrier ED, Shernan SK, Taylor KM, Van de Werf F, Newman MF, Chen JC, et al. Terminal complement blockade with pexelizumab during coronary artery bypass graft surgery requiring cardiopulmonary bypass: a randomized trial. JAMA. 2004;291:2319-27.

7. APEX AMI Investigators; Armstrong PW, Granger CB, Adams PX, Hamm C, Holmes D Jr, et al.Pexelizumab for acute ST-elevation myocardial infarction in patients undergoing primary percutaneous coronary intervention: a randomized controlled trial. JAMA. 2007;297: 43-51.

8. Vakeva AP, Agah A, Rollins SA, Mata LA, Li L, Stahl GL. Myocardial infarction and apoptosis after myocardial ischemia and reperfusion: role of terminal complement components and inhibition by anti-C5 therapy. Circulation. 1998;97:2259-67.

9. Cobb, JP, O'Keefe GE. Injury research in the genomics era. Lancet. 2004;363:2076-83.

10. Collins FS, Green ED, Gottmacher AE, Guyer MS. A vision for the future of genomics research. Nature. 2003;422:835-47. 